\title{
Neutralizing antibodies against the SARS-CoV-2 Omicron variant BA.1 following homologous and heterologous CoronaVac or BNT162b2 vaccination
}

\author{
Samuel M. S. Cheng ${ }^{1,9}$, Chris Ka Pun Mok ${ }^{2,3,9}$, Yonna W. Y. Leung ${ }^{1}$, Susanna S. Ng ${ }^{4}$, Karl C. K. Chan ${ }^{1}$,

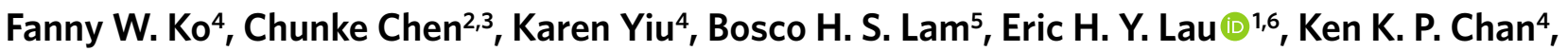

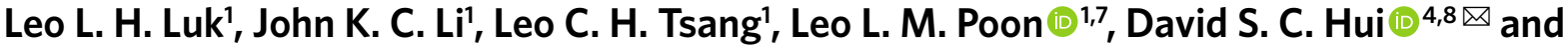 \\ Malik Peiris ${ }^{1,7} \bowtie$
}

The Omicron variant is rapidly becoming the dominant SARS-CoV-2 virus circulating globally. It is important to define reductions in virus neutralizing activity in the serum of convalescent or vaccinated individuals to understand potential loss of protection against infection by Omicron. We previously established that a $\mathbf{5 0 \%}$ plaque reduction neutralization antibody titer (PRNT $_{50}$ ) $\geq \mathbf{2 5 . 6}$ in our live virus assay corresponded to the threshold for $50 \%$ protection from infection against wild-type (WT) SARS-CoV-2. Here we show markedly reduced serum antibody titers against the Omicron variant (geometric mean titer $(\mathrm{GMT})<10)$ compared to WT virus 3-5 weeks after two doses of BNT162b2 $(G M T=218.8)$ or CoronaVac vaccine (GMT = 32.5). A BNT162b2 booster dose elicited Omicron PRNT P $_{50}$ titers $\geq \mathbf{2 5 . 6}$ in $\mathbf{8 8} \%$ of individuals ( 22 of 25) who previously received 2 doses of BNT162b2 and $80 \%$ of individuals (24 of 30 ) who previously received CoronaVac. However, few (3\%) previously infected individuals (1 of 30 ) or those vaccinated with three doses of CoronaVac (1 of 30) met this threshold. Our findings suggest that countries primarily using CoronaVac vaccines should consider messenger RNA vaccine boosters in response to the spread of Omicron. Studies evaluating the effectiveness of different vaccines against the Omicron variant are urgently needed.

A new SARS-CoV-2 variant with increased transmissibility was first reported in South Africa in November 2021 (ref. ${ }^{1}$ ), classified as a variant of concern and named Omicron (BA.1 sublineage of B.1.1.529) (ref. ${ }^{2}$ ). This variant has 37 amino acid substitutions in the spike (S) protein of the virus, 15 of them being in the receptor-binding domain. It was predicted that some of these amino acid substitutions would enable the evasion of neutralizing antibodies. Virus neutralizing antibodies are a major determinant of protection from infection in humans and macaques experimentally challenged with virus ${ }^{3,4}$. Neutralizing antibody thresholds associated with protection from reinfection or severe disease have been reported $^{5,6}$. Although CD8 T cells have been shown to contribute to protection, quantitative correlates of protection are elusive $e^{4}$.
CoronaVac is one of the World Health Organization (WHO)approved vaccines and over two billion doses have been administered in more than 40 countries. Phase 3 randomized clinical trials of CoronaVac showed vaccine efficacy of $50.7 \%$ and higher vaccine effectiveness against severe disease ${ }^{7,8}$. However, breakthrough infections leading to severe disease and death in adults vaccinated with CoronaVac have been reported ${ }^{9}$. Data on the immunogenicity of current COVID-19 vaccines against the Omicron variant is urgently needed.

We previously demonstrated that those vaccinated with BNT162b2 had markedly higher levels of geometric mean PRNT $_{50}$ antibody titers against SARS-CoV-2 isolated in Hong Kong in January 2020 compared to those vaccinated with CoronaVac vaccines at 3-5 weeks postsecond vaccine dose ${ }^{10}$. Allowing for antibody waning, we estimated that only $16 \%$ of individuals vaccinated with CoronaVac would retain $\mathrm{PRNT}_{50}$ antibody titers above protective thresholds against the WT virus while $79.6 \%$ of BNT162b2 vaccinees would by 6 months after the second dose of vaccine ${ }^{10}$. Subsequently, we randomized the cohort receiving CoronaVac vaccine to receive booster doses of CoronaVac or BNT162b2 and showed a marked increase in neutralizing antibodies to WT SARS-CoV-2 after boosting with BNT162b2 but less of an increase with CoronaVac ${ }^{11}$.

In this study, we compare the $\mathrm{PRNT}_{50}$ and $\mathrm{PRNT}_{90}$ GMTs to WT SARS-CoV-2 and its Omicron BA.1 variant in subsets of sera from seven groups of vaccinated individuals, convalescent individuals and individuals with breakthrough infections (Table 1, Extended Data Table 1 and Extended Data Table 2). We evaluated sera from (1) vaccinated individuals with no evidence of previous COVID-19 infection (Methods) 3-5 weeks after receiving two doses of BNT162b2 $(n=31)$ or two doses of CoronaVac $(n=30)$, randomly selected from a previous study ${ }^{10}$, and (2) individuals 3-5 weeks after receiving a third dose of CoronaVac $(n=30)$ or a heterologous booster dose of BNT162b2 after 2 previous doses of CoronaVac $(n=30)$, randomly selected from a previous study (ClinicalTrials.Gov ID NCT04611243) (ref. ${ }^{11}$ ), and (3) those receiving a third dose of BNT162b2 $(n=25)$. We also evaluated sera from

\footnotetext{
'School of Public Health, Li Ka Shing Faculty of Medicine, The University of Hong Kong, Hong Kong, P. R. China. ${ }^{2}$ The Jockey Club School of Public Health and Primary Care, The Chinese University of Hong Kong, Hong Kong, P. R. China. ${ }^{3}$ Li Ka Shing Institute of Health Sciences, Faculty of Medicine, The Chinese University of Hong Kong, Hong Kong, P. R. China. ${ }^{4}$ Department of Medicine \& Therapeutics, Faculty of Medicine, The Chinese University of Hong, Kong, Hong Kong, P. R. China. ${ }^{5}$ Department of Pathology, North Lantau Hospital, Hong Kong, P. R. China. ${ }^{6}$ Laboratory of Data Discovery for Health Limited, Hong Kong Science Park, Hong Kong, P. R. China. ${ }^{7}$ Centre for Immunology and Infection, Hong Kong Science Park, Hong Kong, P. R. China. ${ }^{8}$ Stanley Ho Centre for Emerging Infectious Diseases, Faculty of Medicine, The Chinese University of Hong Kong, Hong Kong, P. R. China. ${ }^{9}$ These authors contributed equally: Samuel M. S. Cheng, Chris Ka Pun Mok.凶e-mail: dschui@cuhk.edu.hk; malik@hku.hk
} 
Table 1 | Age, sex and geometric mean PRNT $_{50}$ titers versus WT or Omicron variant BA.1 3-5 weeks postvaccination

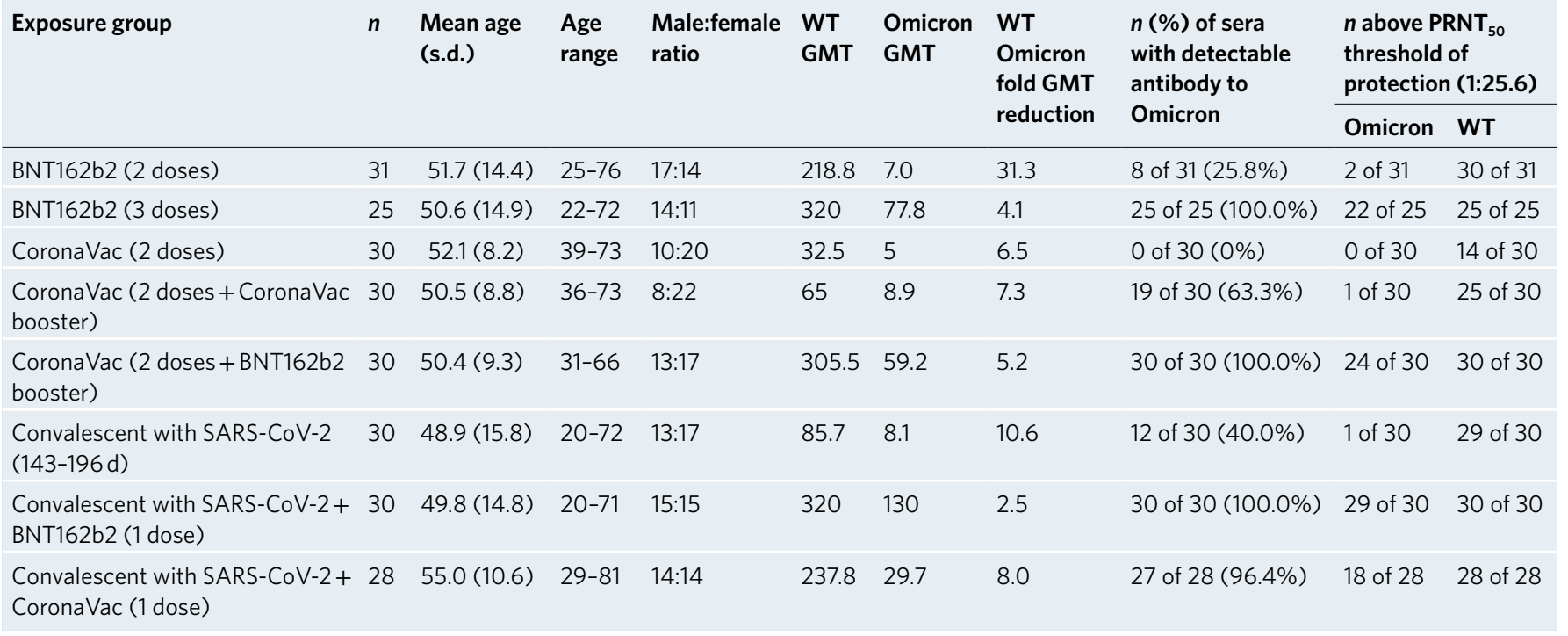

the following groups of previously infected individuals: (1) individuals 143-196 d postinfection who had recovered from COVID19 (pre-Omicron emergence) and had not yet received a vaccine $(n=30)^{12},(2)$ convalescent individuals with COVID-19 who had received one dose of BNT162b2 $(n=30)$ or $(3)$ convalescent individuals with COVID-19 who had received one dose of CoronaVac $(n=28)$. Sera collected during acute infection and convalescence from six Omicron-infected individuals identified in Hong Kong in November and December 2021 were also profiled. Plaque reduction neutralization tests were carried out as described previously (Methods) ${ }^{12,13}$. The highest serum dilutions neutralizing $\geq 50 \%$ or $\geq 90 \%$ of plaques were regarded as the $\mathrm{PRNT}_{50}$ and $\mathrm{PRNT}_{90}$, respectively. SARS-CoV-2 viruses used were a WT virus isolated in January 2020, an Omicron variant isolated on 13 November 2021 and a Delta lineage virus isolated in June 2021.

The $\mathrm{PRNT}_{50}$ titers with the WT SARS-CoV-2 or Omicron variant in these cohorts are shown in Fig. 1. The $\mathrm{PRNT}_{90}$ data are shown in Extended Data Fig. 1. The PRNT ${ }_{50}$ titers in all COVID-19-vaccinated or convalescent cohorts were markedly lower against the Omicron variant than against the WT virus (Fig. 1a and Table 1). Relative to GMT against WT virus, there was a 31-fold reduction after two doses of BNT162b2 vaccine, a 6.5-fold reduction after two doses of CoronaVac vaccine and a 10.6-fold reduction in COVID-19 convalescent sera (Fig. 1 and Table 1). Other studies reported comparable reductions in sera neutralizing titers in messenger RNA-vaccinated or convalescent individuals with COVID-19 (refs. ${ }^{14,15}$ ). However, it is important to note that comparison of fold difference in GMT to the two viruses is confounded when some vaccine groups have low neutralizing antibody GMT to the WT virus because only a smaller fold reduction is possible before titers reach the detection threshold. Booster doses of BNT162b2 and CoronaVac vaccines increased the Omicron PRNT $_{50}$ GMT from 7 to 77.8 and from 5 to 8.9, respectively; however; CoronaVac vaccinees boosted with BNT162b2 had a GMT of 59.2. Convalescent individuals with COVID-19 had an Omicron GMT of 8.1 and one dose of BNT162b2 or CoronaVac increased these GMT to 130 and 29.7, respectively.

Using methods described by Khoury et al. ${ }^{5}$, we previously determined that the threshold for $50 \%$ protection from symptomatic WT SARS-CoV-2 infection was a $\mathrm{PRNT}_{50}$ titer of 25.6 with a $95 \%$ confidence interval $(\mathrm{CI})=18.3-36.0$ (ref. ${ }^{12}$ ). Using this threshold for protection, we observed that 30 of 31 individuals vaccinated with BNT162b2 retained protective $\mathrm{PRNT}_{50}$ antibody titers to WT virus at $3-5$ weeks after the second BNT162b2 dose but only 2 in 31 met this threshold with the Omicron variant. None of those vaccinated with CoronaVac met this protection threshold with the Omicron variant (Table 1). A homologous booster dose of BNT162b2 in individuals previously vaccinated with $\mathrm{BNT} 162 \mathrm{~b} 2$ restored protective thresholds of Omicron variant $\mathrm{PRNT}_{50}$ antibody in 22 of 25 vaccinees but a homologous booster dose of Corona Vac in CoronaVac vaccinees did not (1 in 30). However, after a heterologous BNT162b2 booster, 24 of 30 individuals who previously received 2 doses of CoronaVac had protective levels of $\mathrm{PRNT}_{50}$ antibodies to the Omicron variant. In previously infected individuals who were subsequently vaccinated with 1 dose of either BNT162b2 or CoronaVac, 29 of 30 and 18 of 28 , respectively had protective levels of $\mathrm{PRNT}_{50}$ antibodies to the Omicron variant 3-5 weeks after the vaccine dose (Table 1 ).

Neutralizing antibodies were reduced by 4.7 -fold within $5-5.8$ months after the second dose of BNT162b2 vaccine and declined 6.4-fold within 6 months after two doses of CoronaVac vaccine ${ }^{16,17}$. It is not clear if antibody waning is similar after a booster dose nor whether waning of cross-neutralization antibodies against Omicron would follow similar kinetics. There is, however, evidence that waning of protection against the Delta variant after a booster dose of BNT162b2 is relatively rapid ${ }^{18}$. Allowing for a hypothetical 4.7-fold decline of $\mathrm{PRNT}_{50}$ antibody levels to the Omicron variant, we estimated that 7 of $25(28 \%)$ of those vaccinated and boosted with BTN162b2, and 8 of $30(27 \%)$ individuals vaccinated with CoronaVac and boosted with BNT162b2 would retain $\mathrm{PRNT}_{50}$ antibody levels above this threshold. However, 0 of 30 (0\%) of individuals who received 3 doses of CoronaVac would.

Our convalescent cohort with COVID-19 was already 4.8-6.5 months postsymptom onset and only 1 of 30 individuals met the protective antibody threshold for the Omicron variant. Vaccinating convalescent individuals with COVID-19 with one dose of BNT162b2 or CoronaVac vaccine boosted $\mathrm{PRNT}_{50}$ antibody levels to above the protective threshold against Omicron in 29 of 30 (97\%) and 18 of $28(64 \%)$ individuals, respectively at 3-5 weeks postvaccination. However, the levels achieved in individuals vaccinated with CoronaVac $(\mathrm{GMT}=29.7)$ was lower than for BNT162b2 $(\mathrm{GMT}=130)$; assuming a 4.7 -fold reduction in antibodies, only 2 of 28 would have $\mathrm{PRNT}_{50}$ titers $>25.6$ by $5-5.8$ months postvaccination, although 19 of 30 of those receiving BNT162b2 would.

Six individuals with travel-associated vaccine breakthrough Omicron variant infection were also studied, three with mildly symptomatic illness (cases B, D, E) and three with asymptomatic infection (cases A, C, F). Five were males and 1 female, ranging 

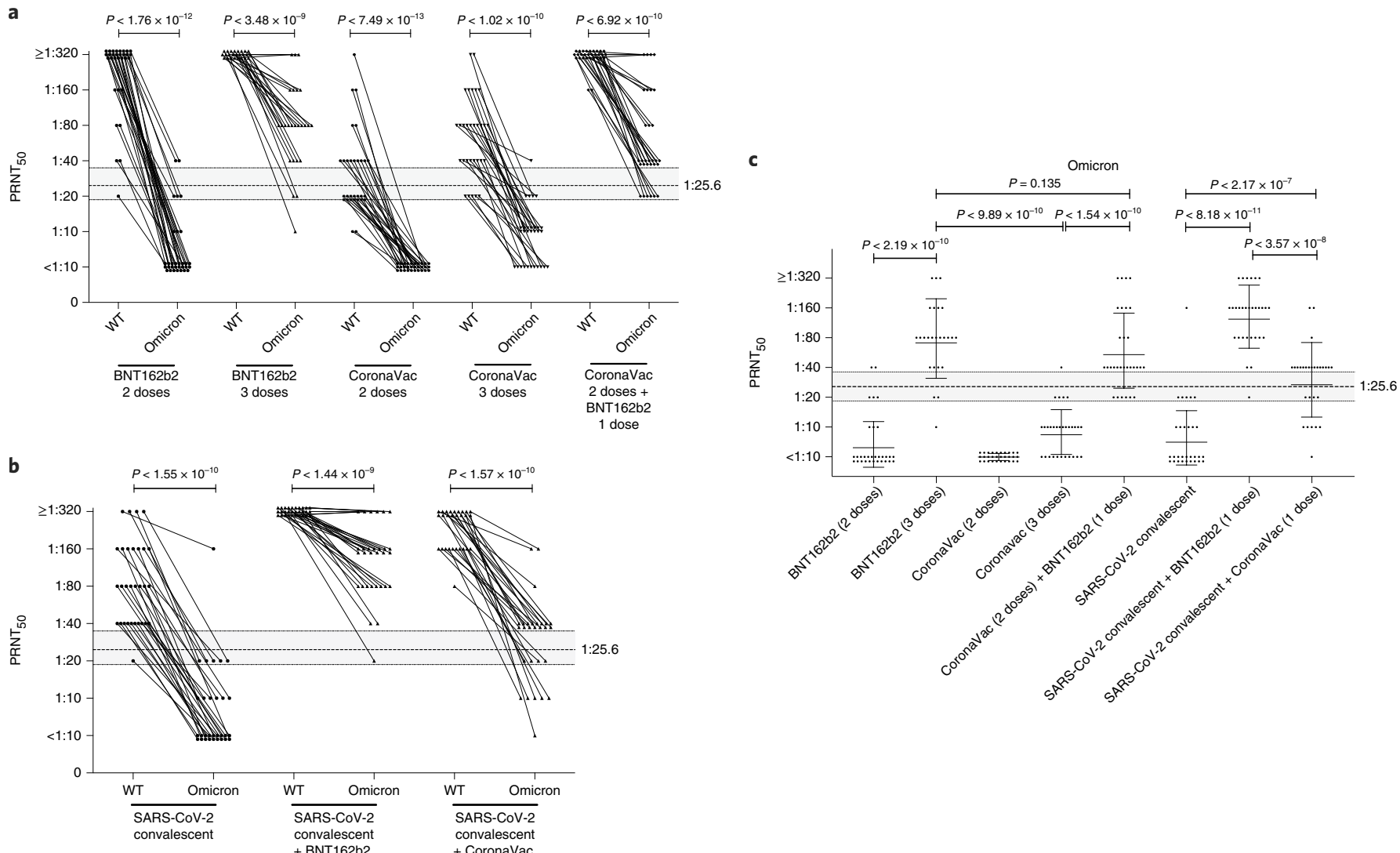

Fig. 1 PRNT $_{50}$ antibody titers to WT virus and Omicron variant BA.1. a, Individuals given two or three doses of BNT162b2 or CoronaVac vaccines, as

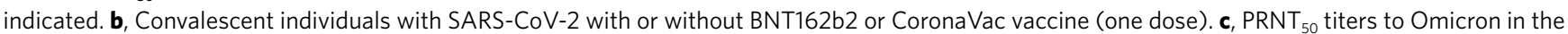
different groups with a geometric mean \pm s.d. are denoted. See Table 1 for the number of individuals in each group. A Mann-Whitney U-test (two-tailed) was used for significance testing. $P$ values for each comparison are denoted. The dotted line indicates the PRNT so $_{0}$ threshold of protection and the shading indicates the $95 \% \mathrm{Cls}$.

in age from 22 to 62 years, and were vaccinated with two doses of BNT162b2 $(n=4)$, mRNA-1273 $(n=1)$ and CoronaVac $(n=1)$ (Extended Data Table 2). Five of them had a first serum sample collected within $1 \mathrm{~d}$ of PCR with reverse transcription diagnosis with Omicron infection and they all had Omicron $\mathrm{PRNT}_{50}$ titers of 10 or lower, possibly explaining why they had Omicron breakthrough infections even though $3(\mathrm{~A}, \mathrm{D}, \mathrm{F})$ had a $\mathrm{PRNT}_{50}$ of $80-160$ to the WT virus (Extended Data Fig. 2). All five showed a rise in antibody titers to Omicron and the WT and Delta viruses. None of these individuals were previously diagnosed with SARS-CoV-2 infection. Acquiring Delta in infection in Hong Kong is unlikely since there have been only six locally acquired Delta infections in Hong Kong in 2021. The duration of travel away from Hong Kong for 5 of these individuals was 18-31 d (Extended Data Table 2) and it is unlikely that they recovered from Delta and got reinfected with Omicron within that time frame. Three of them $(\mathrm{A}, \mathrm{D}, \mathrm{F})$ had higher titers to WT than to Delta in the acute serum, which is more compatible with vaccine immunity than past infection with the Delta variant. The consistent finding of boosting of antibody responses to the Delta virus in these individuals may indicate that Omicron infection in people vaccinated with mRNA or inactivated vaccines may lead to broadening immunity to a range of virus variants, even ones they have not been previously exposed to. A similar finding of increased neutralizing antibodies to the Delta variant in previously vaccinated individuals with Omicron infection has been reported by others ${ }^{19}$.

This study has some limitations. There were relatively small numbers in the individual groups, but as estimated from our power calculations, the sample size was adequate to demonstrate significant difference in neutralizing antibody responses between the groups studied. We have not investigated other antibody activities, such as binding to the $S$ protein or binding to Fc receptors, which may enable antibody-dependent cell cytotoxicity. In a previous study with cohorts vaccinated with two doses of BNT-162b2 or two doses of CoronaVac, we showed that both S protein and FcgRIIIa binding antibodies correlated with the PRNT antibody responses ${ }^{10}$. However, it is possible that these non-neutralizing antibodies may play more important roles in protection from Omicron because they may be less affected by mutations in the $S$ protein found in the Omicron variant virus. We have also not investigated $\mathrm{T}$ cell responses elicited by these vaccines, which may provide protection.

In summary, we demonstrate that two doses of BNT162b2 or CoronaVac vaccine elicit low neutralizing antibodies against the Omicron variant, even at 3-5 weeks postvaccination. Homologous or heterologous BNT162b2 booster doses after two doses of either BNT162b2 or CoronaVac improve neutralizing antibody levels against the Omicron variant at 3-5 weeks postbooster dose. Three doses of CoronaVac failed to elicit neutralizing antibody responses to Omicron in most recipients. Our findings suggest that countries that primarily use CoronaVac vaccines may need to consider mRNA vaccine boosters in response to the spread of Omicron. However, CoronaVac has previously been shown to elicit a wider range of virus-specific $\mathrm{T}$ cell responses, which may compensate for some loss of neutralizing antibody protection ${ }^{10}$. Immunogenicity studies provide rapid assessment of potential protective effects of vaccines against new variants but vaccine effectiveness studies in the field are urgently required. 


\section{Online content}

Any methods, additional references, Nature Research reporting summaries, source data, extended data, supplementary information, acknowledgements, peer review information; details of author contributions and competing interests; and statements of data and code availability are available at https://doi.org/10.1038/ s41591-022-01704-7.

Received: 27 December 2021; Accepted: 18 January 2022; Published online: 20 January 2022

\section{References}

1. Viana, R. et al. Rapid epidemic expansion of the SARS-CoV-2 Omicron variant in southern Africa. Nature (in the press). https://www.nature.com/ articles/s41586-022-04411-y_reference.pdf

2. World Health Organization. Classification of Omicron (B.1.1.529): SARS-CoV-2 variant of concern 2021; https://www.who.int/news/item/26-11-2021classification-of-omicron-(b.1.1.529)-sars-cov-2-variant-of-concern

3. Addetia, A. et al. Neutralizing antibodies correlate with protection from SARS-CoV-2 in humans during a fishery vessel outbreak with a high attack rate. J. Clin. Microbiol. 58, e02107-20 (2020).

4. McMahan, K. et al. Correlates of protection against SARS-CoV-2 in rhesus macaques. Nature 590, 630-634 (2021)

5. Khoury, D. S. et al. Neutralizing antibody levels are highly predictive of immune protection from symptomatic SARS-CoV-2 infection. Nat. Med. 27, 1205-1211 (2021).

6. Cromer, D. et al. Neutralising antibody titres as predictors of protection against SARS-CoV-2 variants and the impact of boosting: a meta-analysis. Lancet Microbe 3, E52-E61 (2022).

7. Zhang, Y. et al. Safety, tolerability, and immunogenicity of an inactivated SARS-CoV-2 vaccine in healthy adults aged 18-59 years: a randomised, double-blind, placebo-controlled, phase 1/2 clinical trial. Lancet Infect. Dis. 21, 181-192 (2021).

8. Jara, A. et al. Effectiveness of an inactivated SARS-CoV-2 vaccine in Chile. $N$. Engl. J. Med. 385, 875-884 (2021).
9. Hundreds of vaccinated Indonesian health workers infected. Bangkok Post (17 June 2021); https://www.bangkokpost.com/world/2133987/ hundreds-of-vaccinated-indonesian-health-workers-infected

10. Mok, C. K. P. et al. Comparison of the immunogenicity of BNT162b2 and CoronaVac COVID-19 vaccines in Hong Kong. Respirology. https://doi. org/10.1111/resp.14191 (2021).

11. Mok, C. K. P. et al. A RCT using CoronaVac or BNT162b2 vaccine as a third dose in adults vaccinated with two doses of CoronaVac. Am. J. Resp. Crit. Care Med. https://doi.org/10.1164/rccm.202111-2655LE (2022).

12. Lau, E. H. et al. Long-term persistence of SARS-CoV-2 neutralizing antibody responses after infection and estimates of the duration of protection. EClinicalMedicine 41, 101174 (2021).

13. Matsuyama, S. et al. Enhanced isolation of SARS-CoV-2 by TMPRSS2-expressing cells. Proc. Natl Acad. Sci. USA 117, 7001-7003 (2020).

14. Cele, S. et al. SARS-CoV-2 Omicron has extensive but incomplete escape of Pfizer BNT162b2 elicited neutralization and requires ACE2 for infection. Nature https://doi.org/10.1038/s41586-021-04387-1 (2021).

15. Schmidt, F. et al. Plasma neutralization of the SARS-CoV-2 Omicron variant. N. Engl. J. Med. https://doi.org/10.1056/NEJMc2119641 (2021).

16. Levin, E. G. et al. Waning immune humoral response to BNT162b2 Covid-19 vaccine over 6 months. N. Engl. J. Med. 385, e84 (2021).

17. Zeng, G. et al. Immunogenicity and safety of a third dose of CoronaVac, and immune persistence of a two-dose schedule, in healthy adults: interim results from two single-centre, double-blind, randomised, placebo-controlled phase 2 clinical trials. Lancet Infect. Dis. 7, https://doi.org/10.1016/S14733099(21)00681-2 (2021).

18. Levine-Tiefenbrun, M. et al. Waning of SARS-CoV-2 booster viral-load reduction effectiveness. Preprint at medRxiv https://doi. org/10.1101/2021.12.27.21268424 (2021).

19. Khan, K. et al. Omicron infection enhances neutralizing immunity against the Delta variant. Preprint at medRxiv https://doi. org/10.1101/2021.12.27.21268439 (2021).

Publisher's note Springer Nature remains neutral with regard to jurisdictional claims in published maps and institutional affiliations.

(C) The Author(s), under exclusive licence to Springer Nature America, Inc. 2022 


\section{Methods}

Clinical specimens. Sera were randomly selected from two previously studied cohorts to investigate the immunogenicity of two doses ${ }^{10}$ or booster doses ${ }^{11}$ of COVID-19 vaccines. The first study had sera collected 3-5 weeks after the second dose of CoronaVac (28 d between the first and second dose) or BNT162b2 vaccine $(21 \mathrm{~d} \text { between first and second dose })^{10}$. The second cohort had sera collected 3-5 weeks after booster vaccination of individuals vaccinated with CoronaVac who were given a third dose of CoronaVac (the interval between the second and third vaccine dose was $61-160 \mathrm{~d} ; 17$ of them were also included in the previous two-dose CoronaVac study) or BNT162b2 (interval between the second and third vaccine dose was $51-141 \mathrm{~d}$; 9 of them were also included in the previous two-dose CoronaVac study ${ }^{11}$. A third group consisted of individuals vaccinated with BNT162b2 receiving a third dose of BNT162b2 (180-234 d after the second dose; 4 of them were included in the previous two-dose BNT162b2 study) with serum collected 3-5 weeks after the third vaccine dose. None of these individuals had a previous history of SARS-CoV-2 infection confirmed with negative IgG to SARS-CoV-2 receptor-binding domain antibody ${ }^{15}$ in the prefirst vaccine dose serum.

Separately, sera were obtained from an ongoing longitudinal study of virologically confirmed convalescent individuals with SARS-CoV-2. Sera from unvaccinated convalescent individuals with COVID-19 were selected from 143 to $196 \mathrm{~d}$ (4.7-6.5 months) postinfection to represent waning antibody titers in convalescence $(n=30)$ ( ref. $\left.^{12}\right)$. Sera were also collected 3-5 weeks postvaccination from convalescent individuals with COVID-19 who received either the BNT162b2 $(n=30)$ or CoronaVac $(n=28)$ vaccine.

These studies were carried out in Hong Kong during the period from 21 February 2020 to 20 November 2021 and was approved by the Joint Chinese University of Hong Kong-New Territories East Cluster Clinical Research Ethics Committee (ref. no. 2020.229). All participants provided informed written consent. The sample sizes for each group are indicated in Table 1. See the statistical analysis for how the sample size was calculated.

Sequential paired sera from six patients diagnosed with Omicron variant infection in November-December 2021 were included as positive controls for comparison purposes (see Extended Data Table 2 for the clinical details).

Virology. Vero-E6 cells (CRL-1586; ATCC) and Vero E6 cells overexpressing transmembrane protease serine 2 (TMPRSS2) (provided by S Matsuyama and colleagues) ${ }^{13}$ were used. Cells were maintained in DMEM medium (Thermo Fisher Scientific) supplemented with $10 \%$ fetal bovine serum (Thermo Fisher Scientific) and $100 \mathrm{U} \mathrm{ml}^{-1}$ of penicillin-streptomycin (Thermo Fisher Scientific). The cells used in the study were regularly tested for Mycoplasma contamination at the Core Facility, Centre for PanorOmic Sciences, University of Hong Kong. We used a strain of the Omicron BA.1 variant isolated from the nose and throat swab of a returning traveler diagnosed with Pango lineage B.1.1.529 Omicron variant infection on 13 November 2021, while in hotel quarantine in Hong Kong. The virus, designated hCoV-19/Hong Kong/VM21044713_WHP5047-S5/2021 was isolated in Vero-E6 TMPRSS2 cells and passage level 3 virus aliquots were used in these studies. The virus had amino acid R346 in the S protein as do most of the Omicron variant viruses to date. The WT SARS-CoV-2 BetaCoV/Hong Kong/VM20001061/2020 isolated in Hong Kong in January 2020 was used for comparison. Delta lineage virus isolated from a clinical specimen collected in June 2021 in Vero-E6 TMPRSS2 cells designated hCoV-19/Hong Kong/21TM280310_HKUVOC0013/2021 was used for comparison. The original clinical swab and the virus isolates were genetically sequenced and it was confirmed that the isolates had an identical amino acid sequence to the virus from the original swab. The virus sequences reported are available in GISAID as EPI_ISL_412028,EPI_ISL_6716902 and EPI_ISL_6716902.

Plaque reduction neutralization tests were carried out in duplicate using 24-well tissue culture plates (TPP Techno Plastic Products) in a biosafety level 3 facility using Vero E6 TMRESS2 cells ${ }^{12,20}$. Serial two-fold dilutions from 1:10 to $1: 320$ of each serum sample were incubated with $30-40$ plaque-forming units of virus for $1 \mathrm{~h}$ at $37^{\circ} \mathrm{C}$. The virus-serum mixtures were added onto preformed cell monolayers and incubated for $1 \mathrm{~h}$ at $37^{\circ} \mathrm{C}$ in a $5 \% \mathrm{CO}_{2}$ incubator. The cell monolayer was then overlaid with $1 \%$ agarose in cell culture medium and incubated for $3 \mathrm{~d}$, at which time the plates were fixed and stained. Antibody titers were defined as the highest serum dilution that resulted in $\geq 90 \%\left(\mathrm{PRNT}_{90}\right)$ or $>50 \%\left(\mathrm{PRNT}_{50}\right)$ reduction in the number of virus plaques. This method has been extensively validated on SARS-CoV-2-infected and control sera previously ${ }^{12,20}$.

The highest serum dilution neutralizing $\geq 50 \%$ and $\geq 90 \%$ of input plaques was regarded as the $\mathrm{PRNT}_{50}$ and $\mathrm{PRNT}_{90}$ titers, respectively. A virus back titration and a positive control serum were included in every experiment. The WHO control serum National Institute for Biological Standards and Control 20/136 was also included in two titrations and gave $\mathrm{PRNT}_{50}$ titers of 20 and 40 with the Omicron virus and 320 and 320 with the WT virus. Virus titers are designated as reciprocal of the serum dilution throughout the text.

Statistical analysis. Sample size calculations. The maximum s.d. of the log titers for the uninfected vaccinated groups were 1.37 and 1.77 for $\mathrm{PRNT}_{50}$ and $\mathrm{PRNT}_{90}$, respectively. Assuming a threefold difference in GMT, a sample size of 10 in each group would have a statistical power $>0.99$ and $>0.94$ for $\mathrm{PRNT}_{50}$ and $\mathrm{PRNT}_{90}$, respectively for detecting a difference between groups using a two-tailed Mann-Whitney $U$-test. Comparisons between groups with a larger sample size or smaller within-group variation would have larger statistical power.

Continuous and categorical variables. Continuous variables were summarized as the geometric mean with s.d. while categorical variables were summarized as proportions or percentages. Sera with undetectable $(<10)$ antibody titers were assigned an antibody titer of 5, for purposes of GMT calculations or statistical comparisons. Comparison between antibody titers to WT and Omicron variant viruses was done using a two-tailed Mann-Whitney $U$-test. Absolute $P$ values were provided. $P<0.05$ was considered statistically significant.

Reporting Summary. Further information on research design is available in the Nature Research Reporting Summary linked to this article.

\section{Data availability}

Source data (individual anonymized patient and linked laboratory data) and raw antibody data are provided with this paper. The viruses used can be obtained on request from Malik Peiris malik@hku.hk. Source data are provided with this paper.

\section{References}

20. Perera, R. A. et al. Serological assays for severe acute respiratory syndrome coronavirus 2 (SARS-CoV-2), March 2020. Euro Surveill. 25, 2000421 (2020).

\section{Acknowledgements}

This research was supported by grants from the Health and Medical Research Fund Commissioned Research on the Novel Coronavirus Disease (COVID-19), Hong Kong SAR (COVID1903003 and COVID190126) (C.K.P.M., D.S.C.H. and M.P.), the National Institutes of Health (grant no. U01-Grant AI151810) (M.P.), National Natural Science Foundation of China/Research Grants Council (RGC) Joint Research Scheme (no. N_HKU737/18) (C.K.P.M. and M.P.), RGC theme-based research schemes (nos. T11-712/19-N and T11-705/21-N) (D.S.C.H., M.P.), Guangdong Province International Scientific and Technological Cooperation Projects (no. 2020A0505100063) (C.K.P.M.), the National Research Foundation (NRF) of Korea grant funded through the Korea government (no. NRF-2018M3A9H4055203) (C.K.P.M.), AIR@InnoHK (E.H.Y.L.) and C2i (L.L.M.P., M.P.) administered by the Innovation and Technology Commission of Hong Kong.

\section{Author contributions}

D.S.C.H., M.P. and C.K.P.M. planned, coordinated and obtained funding for the study and wrote the first draft of the manuscript. S.M.S.C. and L.L.M.P. coordinated the laboratory testing and laboratory data analysis. S.S.N., F.W.K., C.C., K.Y., K.K.P.C. and B.H.S.L. coordinated the clinical studies and data management. Y.W.Y.L., K.C.K.C., L.L.H.L., J.K.C.L. and L.C.H.T. carried out all biosafety level 3 work for the PRNT testing. E.H.Y.L. contributed to the statistical design and analysis. All authors commented on the manuscript draft and agreed to submit the paper for publication.

\section{Competing interests}

The authors declare no competing interests.

\section{Additional information} Extended data is available for this paper at https://doi.org/10.1038/s41591-022-01704-7. Supplementary information The online version contains supplementary material available at https://doi.org/10.1038/s41591-022-01704-7.

Correspondence and requests for materials should be addressed to David S. C. Hui or Malik Peiris.

Peer review information Nature Medicine thanks Deepta Bhattacharya, Ailong Huang and the other, anonymous, reviewer(s) for their contribution to the peer review of this work. Saheli Sadanand was the primary editor on this article and managed its editorial process and peer review in collaboration with the rest of the editorial team.

Reprints and permissions information is available at www.nature.com/reprints. 
A

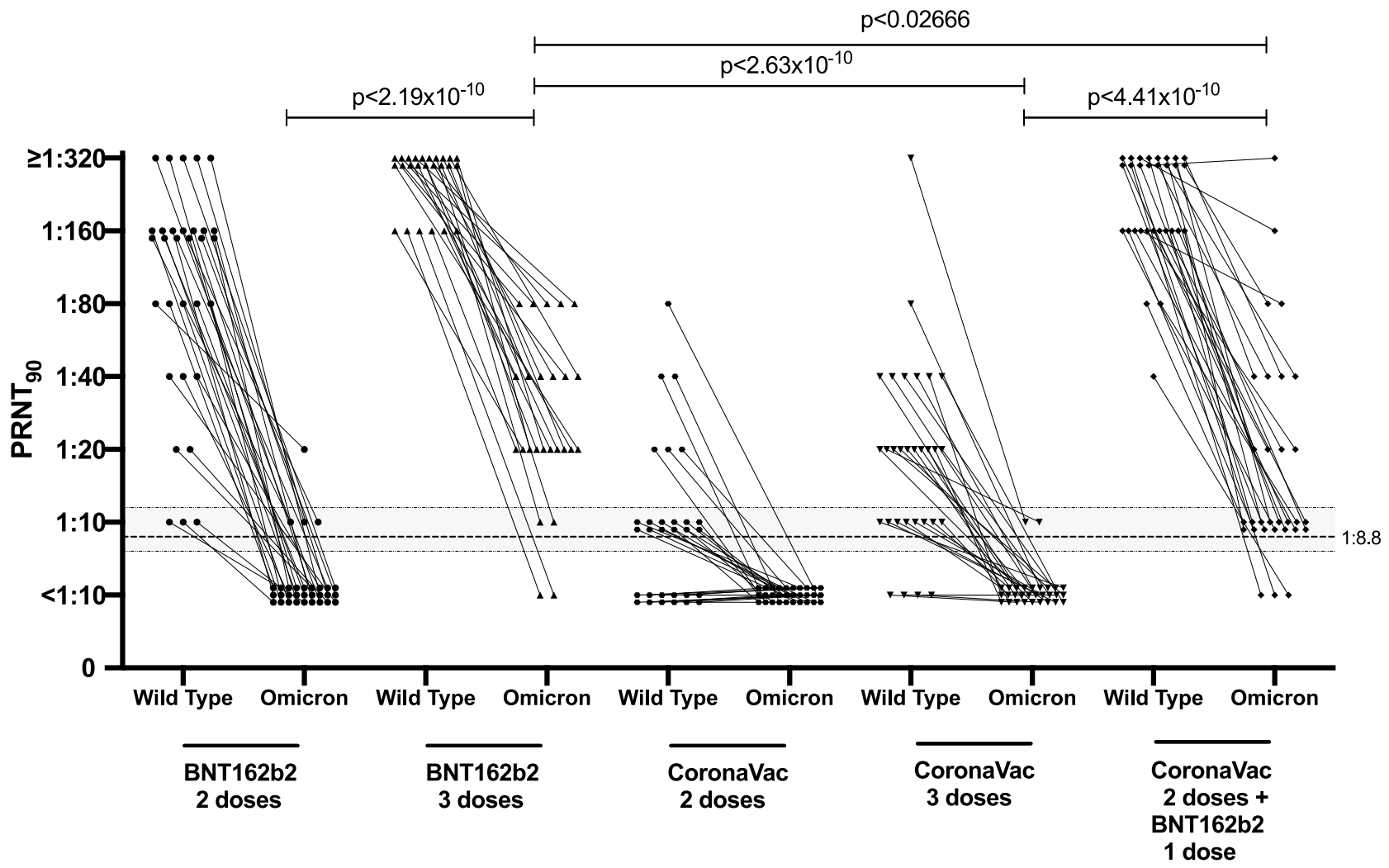

B

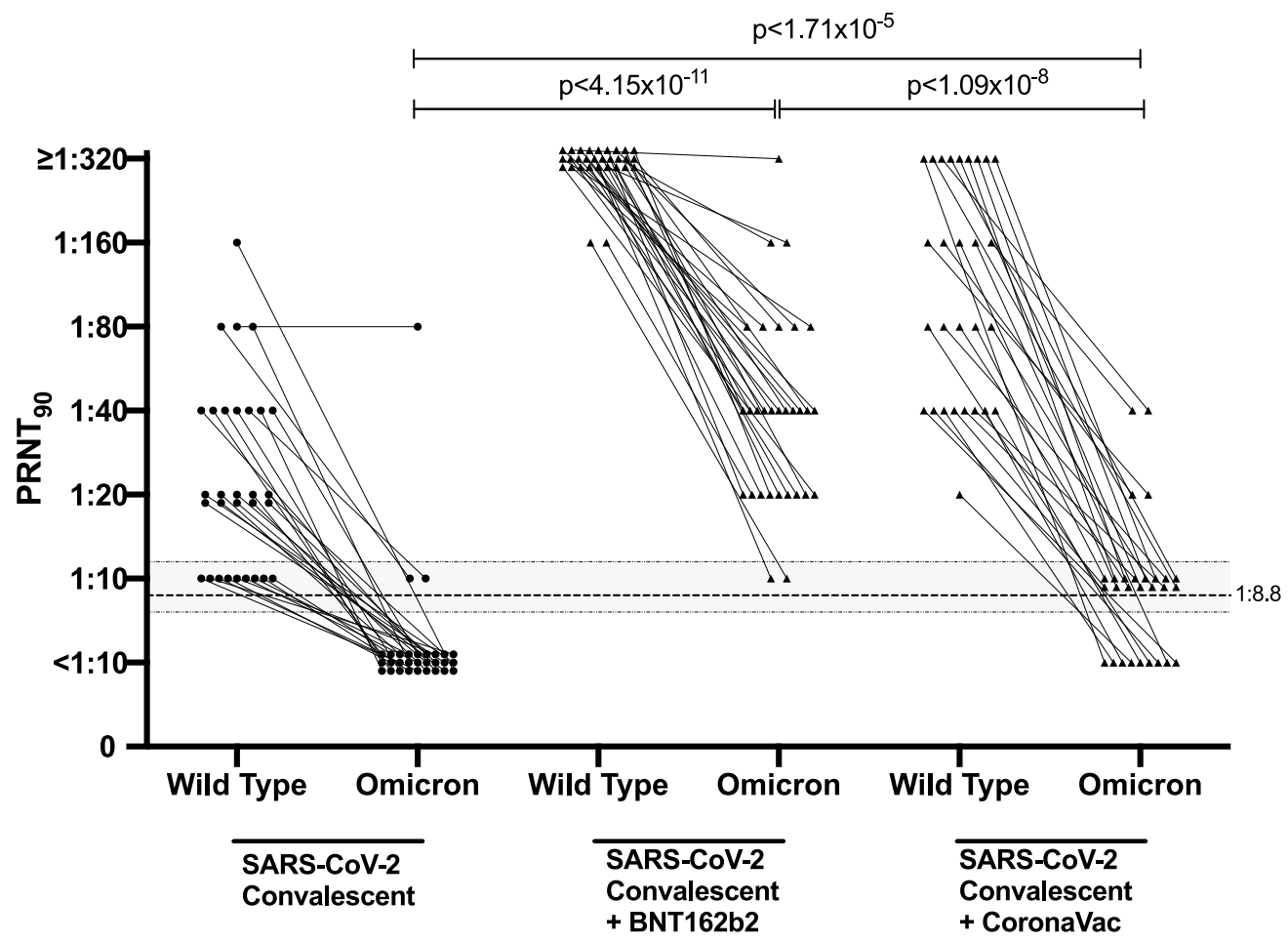

Extended Data Fig. 1 | See next page for caption. 
Extended Data Fig. 1 | 90\% plaque reduction neutralization test (PRNT90) antibody titres to wild-type virus and Omicron variant. $90 \%$ plaque reduction neutralization test (PRNT90) antibody titres to wild-type virus and Omicron BA.1 variant. A. Individuals with 2 or 3 doses of BNT162b2 or CoronVac vaccines, as indicated. B. SARS-CoV-2 convalescent individuals with or without BNT162b2 or CoronaVac vaccine (one dose). See Table for numbers of individuals in each group. Mann-Whitney test (two tailed) was used for significance testing. P values are denoted. Dotted line indicates PRNT90 threshold of protection and shading indicates $95 \%$ confidence intervals (see text). 
Case A vaccinated with BNT162b2

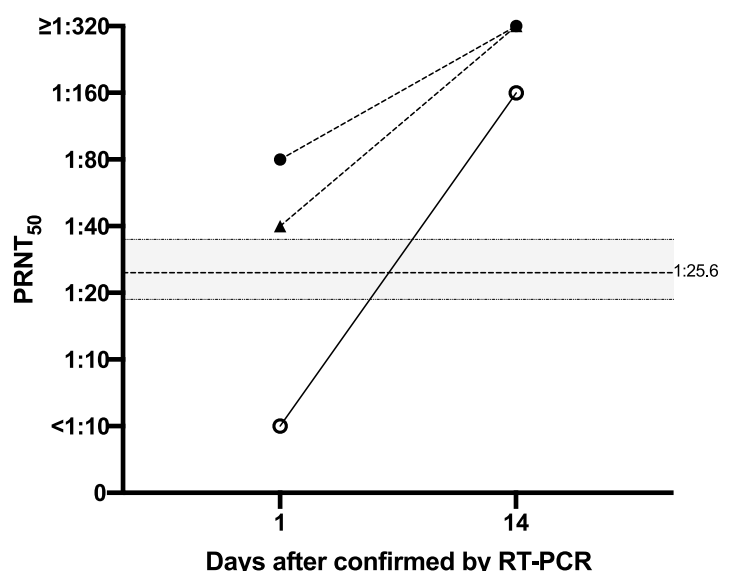

Case C vaccinated with BNT162b2

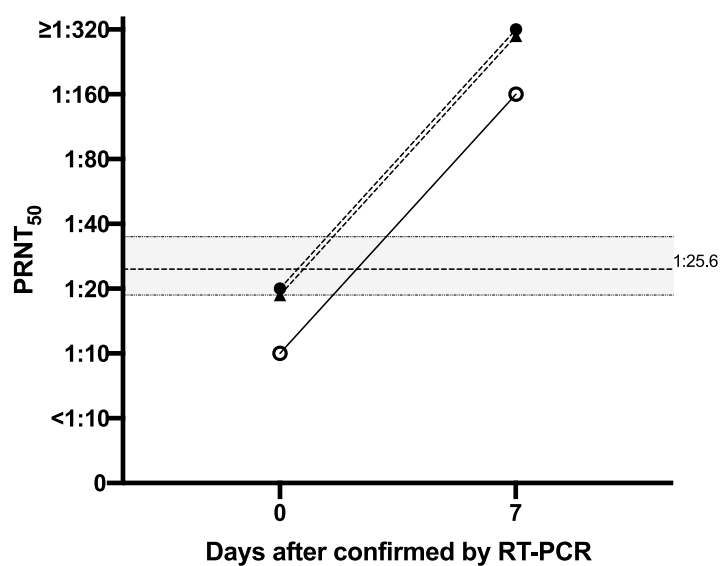

Case E vaccinated with CoronaVac

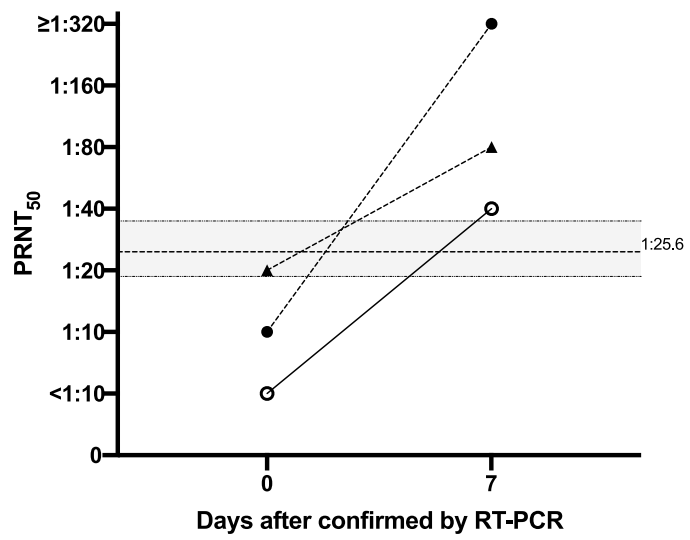

Case B vaccinated with BNT162b2

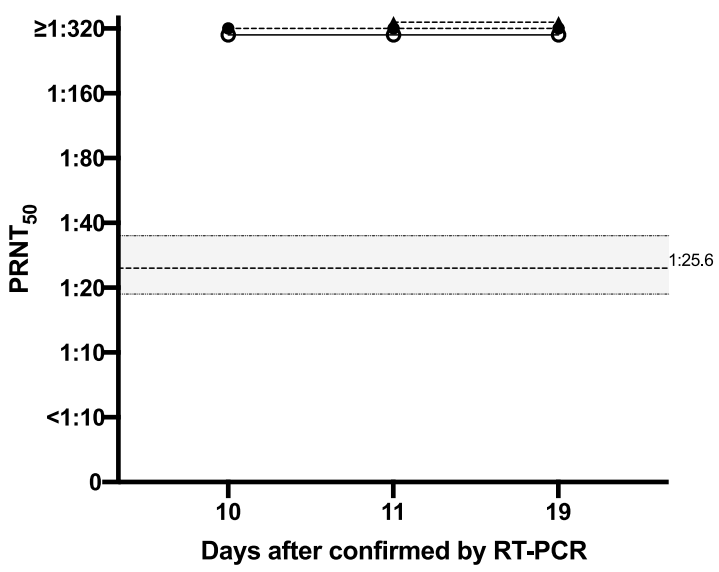

Case D vaccinated with BNT162b2

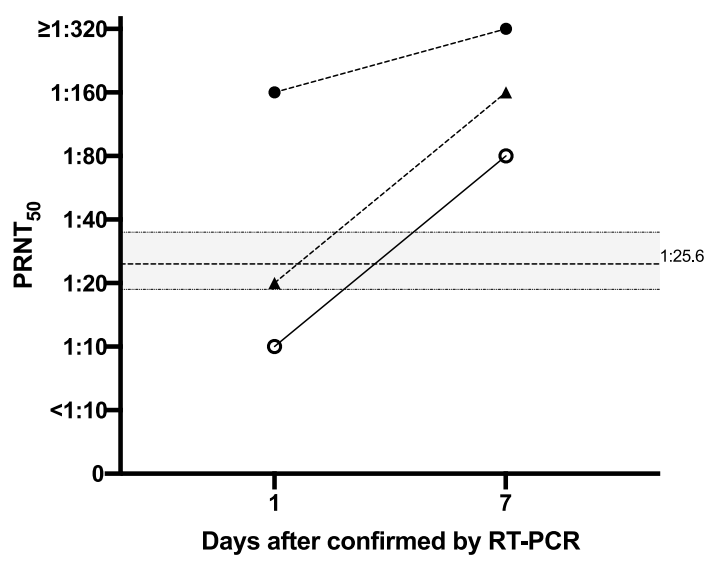

Case $F$ vaccinated with mRNA-1273

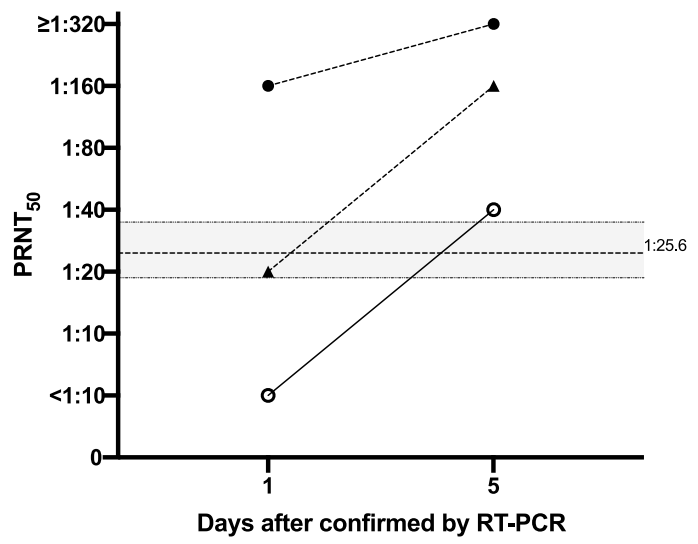

Extended Data Fig. 2 | See next page for caption. 
Extended Data Fig. 2 | 50\% plaque reduction neutralization test (PRNT50) antibody titres to wild type virus, Omicron BA.1 variant and Delta variant in paired sera in six vaccinated individuals with breakthrough Omicron infections. 50\% plaque reduction neutralization test (PRNT50) antibody titres to wild type virus, Omicron variant and Delta variant in paired sera in six vaccinated individuals with breakthrough Omicron infections. The vaccine used in each individual is provided in panel heading and clinical data provided in Extended Data Table 2. Dotted line indicates PRNT50 threshold of protection and shading indicates $95 \%$ confidence intervals (see text). 


\section{NATURE MEDICINE}

Extended Data Table 1 | Comorbidities in the cohorts investigated. Individuals may have more than one comorbidity. The 'other' category includes ischemic heart disease, hyperlipidemia, renal disease, stroke, thrombocytopenia, hepatitis B, hepatitis C, postrenal transplant $(n=1)$, hyperthyroidism, hypothyroidism, asthma, gout, dermatitis, tuberculosis, depression

\begin{tabular}{|c|c|c|c|c|c|c|}
\hline \multirow[t]{2}{*}{ Exposure group } & \multirow[t]{2}{*}{$\mathbf{N}$} & \multicolumn{5}{|c|}{ Co-morbidity } \\
\hline & & None & Diabetes & Hypertension & Others & Not known \\
\hline BNT162b2 3 doses & 25 & 13 & 0 & 3 & 2 & 8 \\
\hline CoronaVac 3 doses & 30 & 20 & 3 & 3 & 8 & 0 \\
\hline CoronaVac 2 doses + BNT162b2 & 30 & 23 & 2 & 2 & 6 & 0 \\
\hline SARS-CoV-2 convalescent & 30 & 11 & 5 & 8 & 19 & 0 \\
\hline $\begin{array}{l}\text { SARS-CoV-2 convalescent + } 1 \\
\text { dose CoronaVac }\end{array}$ & 28 & 14 & 4 & 4 & 14 & 0 \\
\hline
\end{tabular}

Individuals may have more than one co-morbidity.

Other: includes ischemic heart disease, hyperlipidaemia, renal disease, stroke, Thrombocytopenia, hepatitis B, hepatitis $C$, post renal transplant $(\mathrm{n}=1)$, hyperthyroidism, hypothyroidism, asthma, gout, dermatitis, TB, depression. 
Extended Data Table 2 | Clinical information on Omicron infections in returning travelers

\begin{tabular}{|c|c|c|c|c|c|c|c|c|}
\hline $\begin{array}{l}\text { Case } \\
\text { number }\end{array}$ & Age & Sex & $\begin{array}{l}\text { Date of } \\
\text { vaccine } \\
\text { second dose }\end{array}$ & $\begin{array}{l}\text { Type of } \\
\text { vaccine }\end{array}$ & $\begin{array}{l}\text { Date of RT- } \\
\text { PCR } \\
\text { diagnosis }\end{array}$ & $\begin{array}{l}\text { Days since } \\
\text { second dose to } \\
\text { first RT-PCR } \\
\text { positive }\end{array}$ & Symptoms & Period of travel \\
\hline A & 36 & $\mathrm{M}$ & $4 / 6 / 2021$ & BNT162b2 & $13 / 11 / 2021$ & 163 & Asymptomatic & 18 days \\
\hline$B$ & 62 & $\mathrm{M}$ & $25 / 5 / 2021$ & BNT162b2 & $20 / 11 / 2021$ & 187 & Mild & 31 days \\
\hline C & 37 & $\mathrm{M}$ & $25 / 6 / 2021$ & BNT162b2 & $7 / 12 / 2021$ & 165 & Asymptomatic & 19 days \\
\hline D & 22 & $\mathrm{M}$ & $29 / 5 / 2021$ & BNT162b2 & $19 / 12 / 2021$ & 204 & Mild & 18 days \\
\hline$E$ & 28 & $\mathrm{~F}$ & $10 / 2 / 2021$ & CoronaVac & $20 / 12 / 2021$ & 312 & Mild & 20 days \\
\hline $\mathrm{F}$ & 37 & $M$ & $5 / 10 / 2021$ & mRNA-1273 & $26 / 11 / 2021$ & 53 & Asymptomatic & 3 years \\
\hline
\end{tabular}




\section{nature portfolio}

\section{Reporting Summary}

Nature Portfolio wishes to improve the reproducibility of the work that we publish. This form provides structure for consistency and transparency in reporting. For further information on Nature Portfolio policies, see our Editorial Policies and the Editorial Policy Checklist.

\section{Statistics}

For all statistical analyses, confirm that the following items are present in the figure legend, table legend, main text, or Methods section.

$\mathrm{n} / \mathrm{a}$ Confirmed

\ The exact sample size $(n)$ for each experimental group/condition, given as a discrete number and unit of measurement

$\bigotimes$ A statement on whether measurements were taken from distinct samples or whether the same sample was measured repeatedly

$\triangle$ The statistical test(s) used AND whether they are one- or two-sided

Only common tests should be described solely by name; describe more complex techniques in the Methods section.

$\bigotimes$ A description of all covariates tested

\ A description of any assumptions or corrections, such as tests of normality and adjustment for multiple comparisons

$\triangle$ A full description of the statistical parameters including central tendency (e.g. means) or other basic estimates (e.g. regression coefficient)

AND variation (e.g. standard deviation) or associated estimates of uncertainty (e.g. confidence intervals)

For null hypothesis testing, the test statistic (e.g. $F, t, r$ ) with confidence intervals, effect sizes, degrees of freedom and $P$ value noted

Give $P$ values as exact values whenever suitable.

Х $\square$ For Bayesian analysis, information on the choice of priors and Markov chain Monte Carlo settings

$\bigotimes \square$ For hierarchical and complex designs, identification of the appropriate level for tests and full reporting of outcomes

$\bigotimes \square$ Estimates of effect sizes (e.g. Cohen's $d$, Pearson's $r$ ), indicating how they were calculated

Our web collection on statistics for biologists contains articles on many of the points above.

\section{Software and code}

Policy information about availability of computer code

Data collection Not relevant

Data analysis Not relevant

For manuscripts utilizing custom algorithms or software that are central to the research but not yet described in published literature, software must be made available to editors and

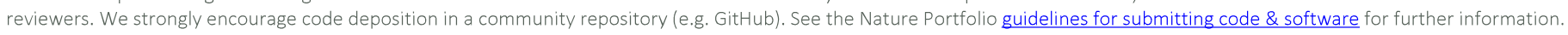

\section{Data}

Policy information about availability of data

All manuscripts must include a data availability statement. This statement should provide the following information, where applicable:

- Accession codes, unique identifiers, or web links for publicly available datasets

- A description of any restrictions on data availability

- For clinical datasets or third party data, please ensure that the statement adheres to our policy 
Please select the one below that is the best fit for your research. If you are not sure, read the appropriate sections before making your selection.

$\bigotimes$ Life sciences $\quad \square$ Behavioural \& social sciences $\quad \square$ Ecological, evolutionary \& environmental sciences

For a reference copy of the document with all sections, see nature.com/documents/nr-reporting-summary-flat.pdf

\section{Life sciences study design}

All studies must disclose on these points even when the disclosure is negative.

$\begin{array}{ll}\text { Sample size } & \text { Sample size based on power calculations and explained in methods (Supplemetnary information). } \\ \text { Data exclusions } & \text { No data were excluded } \\ \text { Replication } & \text { Antibody titrations done in duplicate and mean taken } \\ \text { Randomization } & \begin{array}{l}\text { No randomization was used in the current study. But some samples tested were obtained from a previous randomized clinical study that is in } \\ \text { press. }\end{array} \\ \text { Blinding Those carrying out the serology titrations were blinded to the specimen grouping }\end{array}$

\section{Reporting for specific materials, systems and methods}

We require information from authors about some types of materials, experimental systems and methods used in many studies. Here, indicate whether each material, system or method listed is relevant to your study. If you are not sure if a list item applies to your research, read the appropriate section before selecting a response.

\begin{tabular}{|c|c|}
\hline$n / a$ & Involved in the study \\
\hline Х & $\square$ Antibodies \\
\hline & $\bigotimes$ Eukaryotic cell lines \\
\hline$\bigotimes$ & Palaeontology and archaeology \\
\hline 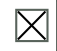 & $\square$ Animals and other organisms \\
\hline & $\bigotimes$ Human research participants \\
\hline 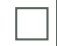 & $\bigotimes$ Clinical data \\
\hline$\bigotimes$ & $\square$ Dual use research of concern \\
\hline
\end{tabular}

\begin{tabular}{|c|c|}
\hline $\mathrm{n} / \mathrm{a}$ & Involved in the study \\
\hline 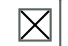 & $\square$ ChIP-seq \\
\hline Х & $\square$ Flow cytometry \\
\hline Х & $\square$ MRI-based neuroimaging \\
\hline
\end{tabular}

\section{Eukaryotic cell lines}

Policy information about cell lines

Cell line source(s)

Authentication

Mycoplasma contamination

Commonly misidentified lines (See $\underline{\text { ICLAC }}$ register)
Obtained from ATCC of Vero-E6 TEMPRSS over-expressing cells were obtained from the authors of cited reference 5. Matsuyama S, et al. Proc Natl Acad Sci U S A. 117:7001-7003 (2020).

As above.

Regular testing of cell lines for mycoplasma was carried out as part of laboratory protocols and described in methods

None

\section{Human research participants}

Policy information about studies involving human research participants

Population characteristics

The population characteristics have been provided in the manuscript

Recruitment

Informed written consent obtained from participants and the clinical study approved by relevant Institutional Review Board. IRB number provided.

Ethics oversight

IRB number provided.

Note that full information on the approval of the study protocol must also be provided in the manuscript. 
Policy information about clinical studies

All manuscripts should comply with the ICMJE guidelines for publication of clinical research and a completed CONSORT checklist must be included with all submissions.

Clinical trial registration ClinicalTrials.gov with identifier NCT04611243

Study protocol

The clinical specimens were drawn from two previous clinical studies, one published and one in press. Both have been cited.

Data collection

Stated in the methods in supplementary information.

Outcomes

Described. 